\title{
Therapeutic Effects of Canagliflozin and Zinc Sulphate Alone and in Combination on Pancreatic Histology in Type-2 Diabetic Rat Model
}

${ }^{1}$ Ayela Eman Zia, ${ }^{1}$ Bushra Suhail, ${ }^{3}$ Zujaja Zaheer

${ }^{I}$ Department of Pharmacology, University College of Medicine and Dentistry, University of Lahore, Lahore

${ }^{2}$ Department of, Pharmacology, King Edward Medical University, Lahore

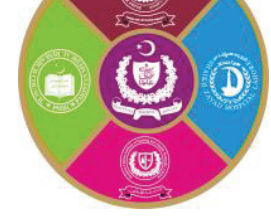

. ABSTRACT
Introduction: Diabetes Mellitus is a common metabolic syndrome characterized by persistently elevated
blood glucose levels. Canagliflozin is an SGLT-2 inhibitor that controls hyperglycemia by reducing the
reabsorption of filtered glucose and excreting it in the urine. Zinc sulphate exhibits some beneficial role in
diabetes mellitus but has not been compared to canagliflozin individually and in combination. Aims \&
Objectives: To observe the effects of treatment with canagliflozin and zinc sulphate on pancreatic histology
in streptozotocin induced type-2 diabetic rat model. Place and duration of study: The study was
conducted in the Department of Pharmacology, KEMU and PGMI, Lahore for the period of two
months. Material \& Methods: It was an animal experimental study of eight weeks duration in which 48
adult healthy albino rats of male gender were divided into six groups and were provided the high fat diet
throughout the study period. Groups A and B were maintained as healthy and diseased controls respectively.
Groups B, C, D, E and F were administered single I/P dose streptozotocin (35mg/kg) at day 22 for inducing
diabetes. Upon confirmation of diabetes after a week the rats were further treated as per group designation
orally for 4 weeks, individually or in combination with full or half doses of canagliflozin (10 mg /kg/day,
5mg/kg/day ) and zinc sulphate (30mg/kg/day, $15 \mathrm{mg} / \mathrm{kg} /$ day). All animals were euthanized at the completion
of study duration. The pancreatic tissue was taken out and examined for the histopathological changes (size
and number of pancreatic islets, karyolysis and ballooning degeneration). Results: There was a marked
improvement in the size and number of islets as well as the inflammatory changes in the combined treatment
group (with canagliflozin in full as well as half dose of zinc sulphate) as compared to the groups given zinc
sulphate and canagliflozin separately. Conclusion: Combined treatment with canagliflozin and zinc sulphate
has a better protective effect on the pancreatic tissue in diabetes than either of them used alone.

Key words: Diabetes mellitus, canagliflozin, zinc sulphate, histopathological, streptozotocin

\section{INTRODUCTION}

$\mathrm{T}$ here has been an alarming increase in the number of individuals having diabetes during the last two decades, which poses a threat to increase the health expenditure for diabetes worldwide and specifically in the developing countries, where the rate of rise is more. ${ }^{1,2}$ The current treatment options provide control of hyperglycemia but the associated side effects worsen the patient's compliance, so there is a dire need of a therapy which can provide a good glycemic control with minimal side effects. ${ }^{3}$ In this regard, canagliflozin, which belongs to the class of SGLT-2 inhibitors seems to be a good option since it is well-tolerated in the body and has additional benefits of lowering blood pressure and body weight. It promotes urinary excretion of glucose via decreasing its reabsorption at the proximal tubule and thus causing a reduction in the renal glucose threshold. ${ }^{4}$ Zinc regulates the functions of many tissues and has the potential to normalize the hyperglycemia. ${ }^{5}$ Limited studies have shown the efficacy of zinc combined with oral hypoglycemic agents in combating diabetes mellitus. ${ }^{6}$ The high-fat diet/streptozotocin (HFD/STZ) rat model is an example of the experimentally induced type 2 diabetes model. It is prepared by combining a fatrich diet to produce insulin resistance and then treating with STZ (a toxin of the beta cells), that leads to greater decrease in the functional mass of the pancreatic beta cells. ${ }^{7}$ 
The present study is designed to observe the role of zinc and canagliflozin alone and in combination on pancreatic histology in type-2 diabetes mellitus and to hypothesize that the combination therapy has a better effect than each given separately.

\section{MATERIAL AND METHODS}

Study Design: Animal experimental study.

Setting: The study was conducted in the Department of Pharmacology, KEMU and PGMI, Lahore.

Sampling Technique: Simple random sampling.

Sample Size: Forty eight rats were divided into six groups by lottery method.

Inclusion Criteria: Male Sprague-Dawley rats, weighing $120 \mathrm{~g}$ to $180 \mathrm{~g}$.

Exclusion Criteria: Rats showing signs of any disease.

48 adult healthy albino rats of male gender were purchased from UVAS (University of Veterinary and Animal Sciences) and kept in the animal house of PGMI (Post-Graduate Medical Institute), Lahore. Animals were divided randomly into 6 equal groups having 8 rats in each group. The rats were exposed to natural day and night cycles at room temperature of $22 \pm 2{ }^{\circ} \mathrm{C}$ with $50 \pm 5 \%$ humidity throughout the experiment. They had a free access to rat chow and water ad libitum. An interval of seven days was given to them to get acclimatized before the start of the experiment.

Preparation of Doses \& Sampling: The calculated dose for individual rat, i.e. $10 \mathrm{mg} / \mathrm{kg} /$ day of canagliflozin $^{8}$ and $30 \mathrm{mg} / \mathrm{kg} /$ day of zinc sulphate ${ }^{9}$ were weighed and dissolved in $1 \mathrm{ml}$ of distilled water. High fat diet was prepared by combining $1.5 \mathrm{~g}$ of cholesterol, $1 \mathrm{~g}$ of sodium deoxycholate and $8 \mathrm{ml}$ of coconut oil in every $100 \mathrm{~g}$ of normal rat chow. ${ }^{10,11}$ Hyperlipidemic rats were then injected intraperitoneally with streptozotocin $(35 \mathrm{mg} / \mathrm{kg})$ at day 22 , dissolved in $0.1 \mathrm{M}$ sodium citrate buffer at $\mathrm{pH} 4.5$ (prepared by adding $46.5 \mathrm{ml}$ of citric acid to $3.5 \mathrm{ml}$ of sodium citrate solution and making it up to $100 \mathrm{ml}$ with distilled water). ${ }^{12}$ After a week from injection of STZ, blood samples obtained from the lateral tail vein were used in determining the blood glucose value using glucometer and those having blood glucose $>180 \mathrm{mg} / \mathrm{dl}$ were labeled as diabetic.

Grouping of animals: Forty eight rats were divided into six groups randomly by lottery method, with 8 rats in each group. These groups were labelled as A,B,C,D,E and F. Each of the group was kept in a separate iron cage. Rats in group A (normal control group) were fed with standard rat diet (normal rat chow), throughout the study period of 8 weeks. Rats in all the other groups $(\mathrm{B}, \mathrm{C}, \mathrm{D}, \mathrm{E}$ and F) were given high fat diet throughout 8 weeks and streptozotocin after 3 weeks for the induction of type-2 diabetes. Rats in group B (disease control group) were only given streptozotocin to induce diabetes. Rats in group $\mathbf{C}$ were administered zinc sulphate orally from $4^{\text {th }}$ to $8^{\text {th }}$ week after the induction of diabetes. Rats in group D were administered canagliflozin orally from $4^{\text {th }}-8^{\text {th }}$ week after the induction of diabetes. Rats in group $\mathbf{E}$ were administered full doses orally of canagliflozin (i.e. $10 \mathrm{mg} / \mathrm{kg} /$ day) and zinc sulphate $(30 \mathrm{mg} / \mathrm{kg} /$ day) from $4^{\text {th }}-8^{\text {th }}$ week after the induction of diabetes. Rats in group $\mathbf{F}$ were administered half doses orally canagliflozin (i.e. $5 \mathrm{mg} / \mathrm{kg} /$ day) and zinc sulphate $(15 \mathrm{mg} / \mathrm{kg} /$ day $)$ from $4^{\text {th }}-8^{\text {th }}$ week after the induction of diabetes. Euthanization: Twenty four hours after last dose administered the rats were sacrificed at the end of week 8. Pancreas of each rat was identified and dissected out en-bloc and preserved in formalin separately.

Histological Examination: After removing the pancreas, it was stretched on filter paper and fixed in $10 \%$ buffered formalin $(\mathrm{pH} 7.4)$ at room temperature. The fixed specimens were sliced, processed and embedded into paraffin blocks. The blocks were then cut into $4 \mu \mathrm{m}$ paraffin sections by a rotator microtome. Clean glass slides were put in hot air oven for 24 hours and were properly numbered and $4 \mu \mathrm{m}$ thick representative sections of tissues were mounted. De-waxing of tissues was done by dipping them in xylene. Slides were hydrated by passing through decreasing concentrations of alcohol and were dipped in hematoxylin. Afterwards, these were washed in the running water. Then, these slides were placed in $1 \%$ acid alcohol, again washed under tap water and put in $1 \%$ ammonia solution followed by water wash. Then, the slides were put in eosin for 5 minutes and washed under tap water. The stained sections were mounted with DPX and examined under microscope to evaluate for the presence or absence of the structural changes like signs of inflammation (karyolysis and ballooning) and atrophy (changes in the size and number of islet of Langerhans). ${ }^{13}$ The size and number of pancreatic islets were measured with the help of a deca-head microscope (Nikon Imaging Software package-D) and mean was taken for further calculations.

\section{Statistical analysis:}

All the data was entered in SPSS version 23 for qualitative data and graph pad prism version 8 for quantitative data. Quantitative data (size and number of islets) was expressed as Mean $\pm \mathrm{S}$. D. 
Mean plots were used for graphical presentation to see the changes in the parameters. The data was evaluated by one way analysis of variance followed by Tukey's multiple comparison tests. Qualitative data (karyolysis and ballooning degeneration) were scored as numbers and expressed as percentage of changes in groups. The significance of differences was measured through Kruskal-Wallis ANOVA and Mann-Whitney U test. p-value of less than 0.05 was considered significant.

\section{RESULTS}

1) Size of Islets of Langerhans $(\mu \mathrm{m})$ :

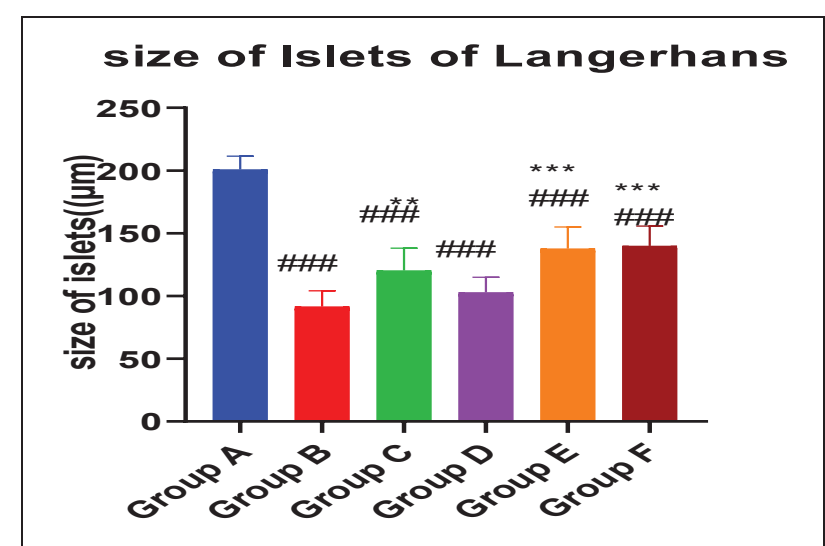

Fig-1: Comparison of mean size of islets of Langerhans of groups A, B, C, D, E and F

\#\#\# indicates p-value is $<0.001$ as compared to group $\mathrm{A}$. *** shows p-value is $<0.001$

** means $\mathrm{p}$ value $<0.01$, when compared to group $\mathrm{B}$.

Group $\mathrm{A}=$ normal control, Group $\mathrm{B}=$ disease control, Group $\mathrm{C}=\mathrm{Zn}$ treated, Group $\mathrm{D}=$ Cana treated, Group $\mathrm{E}=\mathrm{Zn}+$ Cana (full dose), Group $\mathrm{F}=\mathrm{Zn}+\mathrm{Cana}$ (half dose).

\section{2) Number of Islets of Langerhans:}

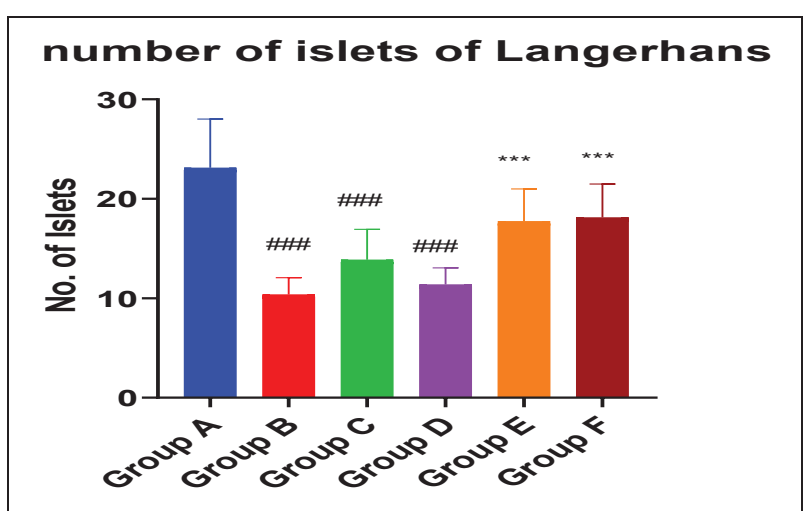

Fig-2: Comparison of mean number of islets of Langerhans among groups A,B,C,D,E and F \#\#\# shows p-value is $<0.001$ when compared to group $\mathrm{A}$. $* * *$ means $\mathrm{p}$-value is $<0.001$ as compared to group $\mathrm{B}$.
Group $\mathrm{A}=$ normal control, Group $\mathrm{B}=$ disease control, Group $\mathrm{C}=\mathrm{Zn}$ treated, Group $\mathrm{D}=$ Cana treated, Group $\mathrm{E}=\mathrm{Zn}+$ Cana (full dose), Group $\mathrm{F}=\mathrm{Zn}+\mathrm{Cana}$ (half dose).

\section{3) Karyolysis:}

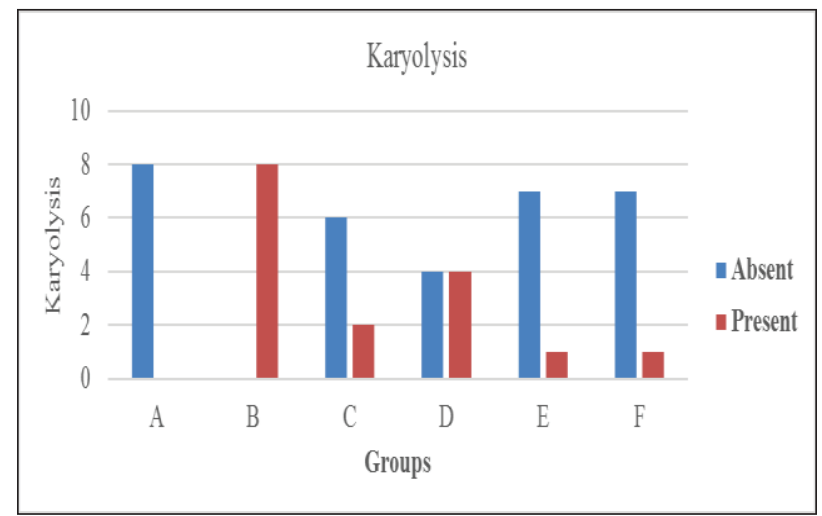

Fig-3: Comparison of karyolysis among groups $\mathrm{A}, \mathrm{B}, \mathrm{C}, \mathrm{D}, \mathrm{E}$ and $\mathrm{F}$.

Group $A=$ normal control, Group $B=$ disease control, Group $C=Z n$ treated, Group $D=$ Cana treated, Group $\mathrm{E}=\mathrm{Zn}+$ Cana (full dose), Group $\mathrm{F}=\mathrm{Zn}+\mathrm{Cana}$ (half dose).

\section{4) Ballooning degeneration:}

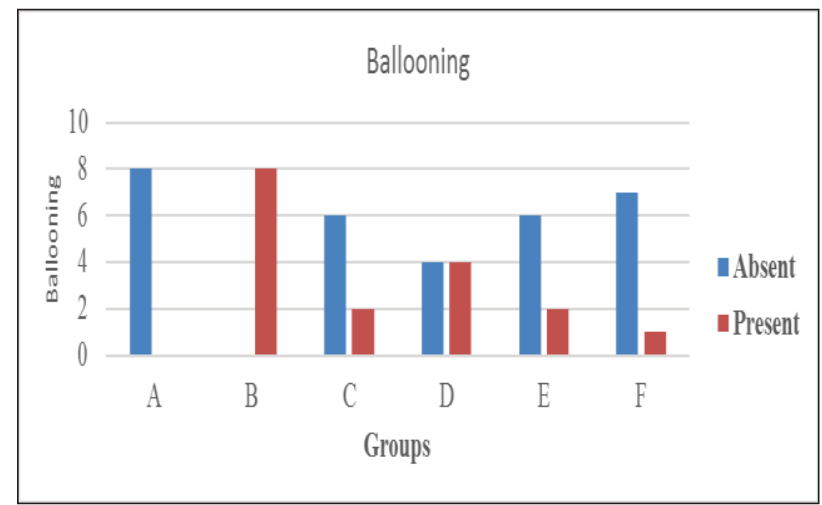

Fig-4: Comparison of ballooning degeneration among groups A, B, C, D, E and F.

Group $\mathrm{A}=$ normal control, Group $\mathrm{B}=$ disease control, Group $\mathrm{C}=\mathrm{Zn}$ treated, Group $\mathrm{D}=$ Cana treated, Group $\mathrm{E}=\mathrm{Zn}+$ Cana (full dose), Group $\mathrm{F}=\mathrm{Zn}+\mathrm{Cana}$ (half dose). 


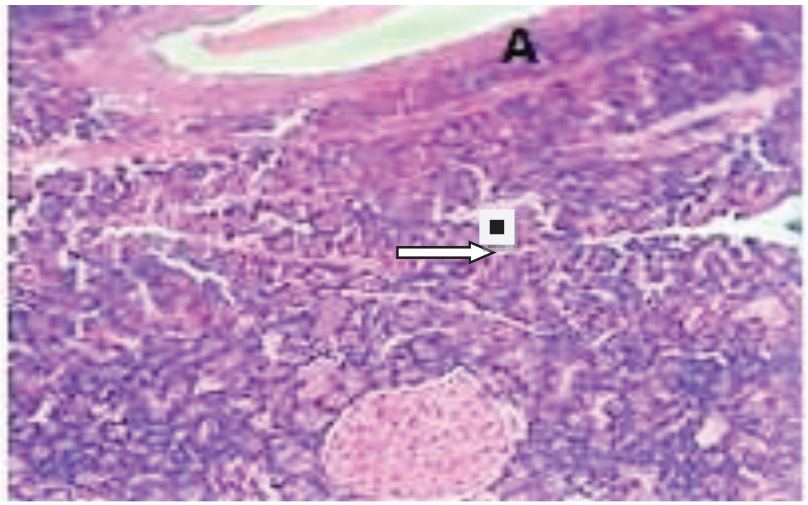

Fig-5: Group A (normal control) Histology of pancreas showing - normal islets of Langerhans $(40 \mathrm{x}$; $\mathrm{H} \& \mathrm{E})$

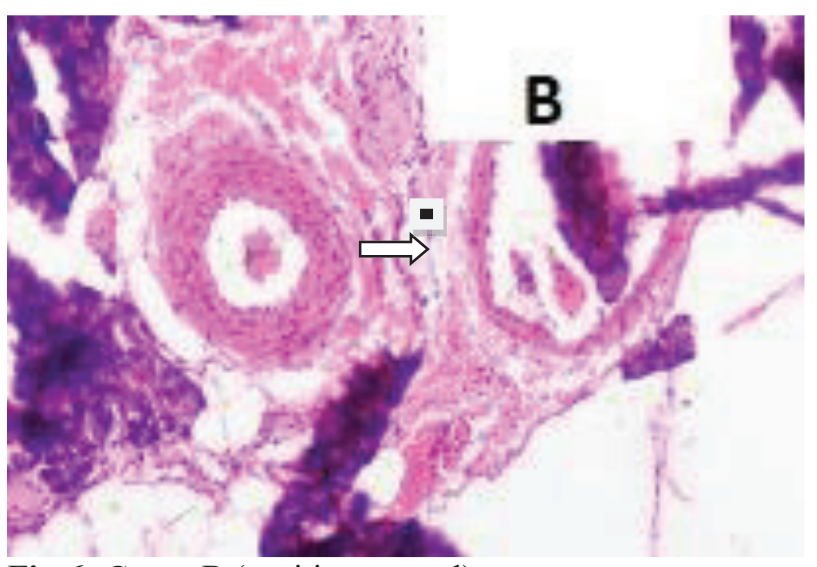

Fig-6: Group B (positive control)

- Severe reduction in the size and number of islets in STZ-induced diabetic rat (40 x; H\&E)

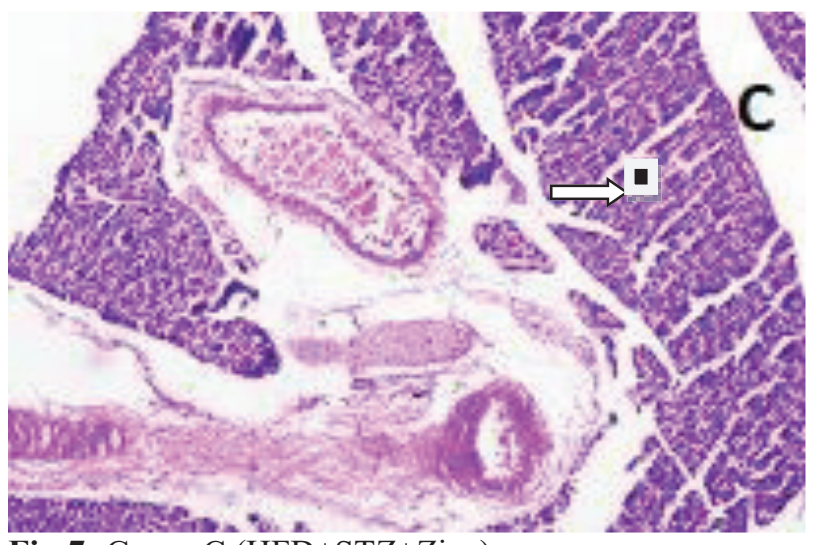

Fig-7: Group C (HFD+STZ+Zinc)

- Some restoration in size and number of islets with moderate karyolysis and ballooning (40 x; H\&E) after treatment with zinc alone

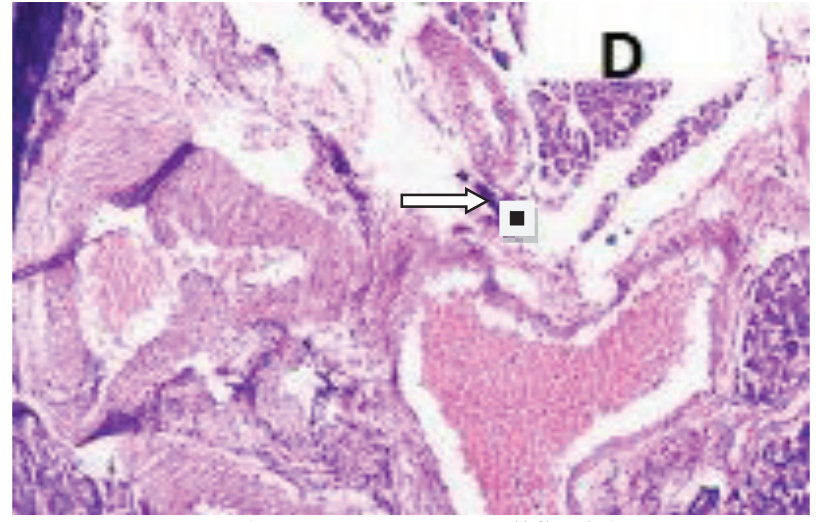

Fig-8: Group D (HFD+STZ+Canagliflozin)

- Some restoration in size and number of islets, ballooning and karyolysis still present after treatment with cana alone (40x; H\&E)

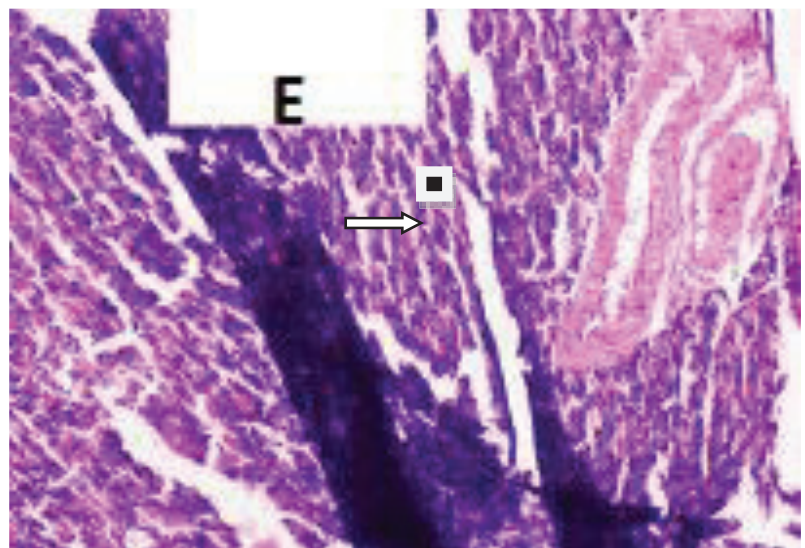

Fig-9: Group E (HFD+STZ+Zinc+full Cana)

- Restoration of size and number of islets with mild karyolysis and ballooning (40x; H\&E)

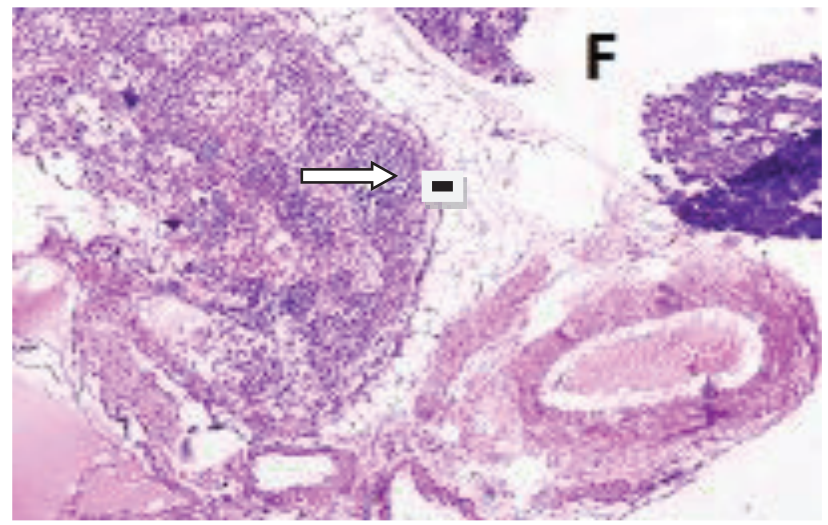

Fig-10: Group F (HFD+STZ+Zinc+halfCana)

- Restoration of the size and number of islets with mild karyolysis and ballooning (40x; H\&E)

\section{DISCUSSION}

Histopathological study with the light and deca-head microscopy revealed some gross changes in the size, number and structure of the islets of Langerhans. STZ administration led to the shrinkage and 
disruption of the structure of islets and the degenerative changes were pronounced, like karyolysis of the nuclei and ballooning degeneration, causing the cells to have a highly vacuolated cytoplasm. Upon injection with STZ, atrophy of islets was prominent causing a visible reduction in the size and number of the islets of Langerhans. Similar findings were reported in the recent works using the rat model of STZ induced diabetes. ${ }^{13,14}$ After the administration of zinc for four weeks, there was some reversal of these histopathological changes in the pancreatic sections of the rats of group C. A significant restoration of the size of islets as well as the reversal of karyolysis and ballooning degeneration was observed on histopathological examination, whereas no significant difference was observed in the number of islets with zinc treatment. These results resemble those of another study in which zinc supplementation given to STZ-induced diabetic rats was associated with significant rejuvenation of the islets histology. ${ }^{5}$ Zinc preserves the pancreatic architecture probably through its anti-oxidant property. ${ }^{15}$ Canagliflozin was also found to exert a beneficial effect in restoring the histological morphology of pancreas (karyolysis and ballooning degeneration) but to a lesser extent than zinc. This observation is in accordance with a study done earlier in which the treatment of ZDF (Zucker Diabetic Fatty) rats with canagliflozin minimized the degenerative changes in diabetic induced islets. ${ }^{16}$ Another study indicated that dapagliflozin (closely related to canagliflozin and both belong to SGLT-2 inhibitors) has a role in the preservation of pancreatic islets morphology of HFD fed diabetic rats ${ }^{17}$. In diabetes, increase in demand of insulin secretion occurs which can cause stress of the endoplasmic reticulum, leading to increased apoptosis of the beta-cell. By decreasing hyperglycemia through a non-insulin-dependent procedure, SGLT-2 inhibitors reduces the demand on the beta cells to produce insulin and thus prevents excessive apoptosis of these cells as seen in diabetes. ${ }^{8,18}$ Even better results were seen in the combination groups $\mathrm{E}$ and $\mathrm{F}$, where the addition of zinc to canagliflozin further improved the morphological changes in the islets. The degenerative signs (karyolysis and ballooning) almost disappeared and the number and size of the pancreatic islets were comparable with the negative control group. No study to date has suggested this potentiating effect of zinc with canagliflozin.

\section{CONCLUSION}

This study has demonstrated that the combined administration of zinc and canagliflozin has exerted a stronger effect on restoring pancreatic histology back to normal in type-2 diabetic rat model as compared to both of these drugs given alone.

\section{REFERENCES}

1. Zimmet PZ, Magliano DJ, Herman WH, Shaw JE. Diabetes: a 21 st century challenge. Lancet Diabetes Endocrinol 2014; 2:56-64.

2. Nanditha A, Ramachandran A, Snehalatha C, Chan JC, Chia KS, Shaw EJ, et al. Diabetes in Asia and the Pacific: Implications for the Global Epidemic. Diabetes care. 2016; 39(3):472-85.

3. Chaudhury A, Duvoor C, Reddy Dendi VS, Kraleti S, Chada A, Ravilla R, et al. Clinical review of antidiabetic drugs: Implications for type 2 diabetes mellitus management. Front Endocrinal. 2017; 8(6):1-12.

4. Brocavich SS, Hilas O. Canagliflozin (Invokana), a novel oral agent for type-2 diabetes. P\&T. 2013; 38(11):656-60, 65-66.

5. Barman S, Srinivasan K. Zinc supplementation alleviates hyperglycemia and associated metabolic abnormalities in streptozotocininduced diabetic rats. Can J Physiol Pharmacol. 2016; 94(12):1356-65.

6. Burki Z, Hussain M, Burki S, Farooqi W, Zeb A, Ahmad S. Effect of zinc supplementation on serum fasting blood sugar and $\mathrm{HbAlc}$ in adult diabetics on oral hypoglycemic agents. Gomal J Med Sci. 2017; 15(1):8-11.

7. Skovso S. Modeling type 2 diabetes in rats using high fat diet and streptozotocin. J Diabetes Investing. 2014; 5(4):349-58.

8. Liang Y, Arakawa K, Ueta K, Matsushita Y, Kuriyama C, Martin T, et al. Effect of canagliflozin on renal threshold for glucose, glycemia, and body weight in normal and diabetic animal models. PloS one. 2012; 7(2):e30555.

9. Nazarizadeh A, Asri-Rezaie S. Comparative study of antidiabetic activity and oxidative stress induced by zinc oxide nanoparticles and zinc sulfate in diabetic rats. AAPS Pharm Sci Tech. 2016; 17(4):834-43.

10. Balasubramanian MN, Muralidharan $P$, Balamurugan G. Anti-hyperlipidemic activity of Pedalium murex (Linn.) fruits on high fat diet fed rats. Int J Pharmacol. 2008; 4(4):310-13. 
11. Desu BSR, Saileela CH. Anti-hyperlipidemic activity of methanolic extract of Rhinacanthus Nasutus. Int J Res Pharm Chem.2013;3(3):708-11

12. Marie M, Arafa N, Sara Abdullah Mubarak A. Effect of canagliflozin or metformin on metabolic disorders in obese diabetic rats. Afr J Pharm Pharmacol. 2015; 9(46):1071-9.

13. Abunasef SK, Amin HA, Abdel-Hamid GA. A histological and immunohistochemical study of betacells in streptozotocin diabetic rats treated with caffeine. Folia Histochem Cytobiol. 2014; 52(1):42-50.

14. Omar NM, Atia GM. Effect of Nigella Sativa on pancreatic $\beta$-cell damage in streptozotocin induced diabetic rats. The Egyptian Journal of Histology. 2012; 35(1):106-16.

15. Zhu K, Nie S, Li C, Huang J, Hu X, Li W, et al. Antidiabetic and pancreas-protective effects of zinc threoninate chelate in diabetic rats may be associated with its antioxidative stress ability. Biol Trace Elem Res. 2013; 153(1-3):291-8.

16. Kuriyama C, Xu JZ, Lee SP, Qi J, Kimata H, Kakimoto T, et al. Analysis of the effect of canagliflozin on renal glucose reabsorption and progression of hyperglycemia in zucker diabetic fatty rats. J Pharmacol Exp Ther. 2014; 351(2):423-31.

17. Macdonald FR, Peel JE, Jones HB, Mayers RM, Westgate L, Whaley JM, et al. The novel sodium glucose transporter 2 inhibitor dapagliflozin sustains pancreatic function and preserves islet morphology in obese, diabetic rats. Diabetes Obes Metab.2010;12(11):1004-12

18. Eizirik DL, Cardozo AK, Cnop M. The role for endoplasmic reticulum stress in diabetes mellitus. Endocr Rev. 2008; 29(1):42-61.

\section{The Authors:}

Dr. Ayela Eman Zia

Senior Demonstrator,

Department of Pharmacology,

UCMD, University of Lahore, Lahore.

Dr. Bushra Suhail

Assistant Professor,

Department of Pharmacology,

UCMD, University of Lahore, Lahore.

Prof. Zujaja Zaheer

Head, Department of Pharmacology,

King Edward Medical University, Lahore.

\section{Corresponding Author:}

Dr. Ayela Eman Zia,

Senior Demonstrator,

Department of Pharmacology,

UCMD, University of Lahore, Lahore.

E-mail: ayelaemanz@gmail.com 